\title{
A Characteristics Measurement of Two-Dimensional Range Scanner and its Application
}

\author{
Takafumi Matsumaru*
}

Bio-Robotics and Human-Mechatronics Lab., Shizuoka Univ., Johoku, Naka, Hamamatsu 432-8561, Japan

\begin{abstract}
This paper shows the result of the characteristics measurement of a two-dimensional active range scanner, URG made by Hokuyo Automatic Co., Ltd. which was released in 2004 and is spreading widely as an external sensor for mobile robot. The following items were clarified from the characteristics measurement of URG-X002S in various conditions. (1) In the case that the object has a gloss surface or a black surface, the error rate (the frequency that the scanner judges to be impossible to measure and an error code is output) rises when the oblique angle of object becomes large and also the distance to object becomes long. (2) In the case that the object has a white surface or a rough surface, not only the error rate is zero but also the margin of error becomes dozens of millimeters and the varying is small, if the oblique angle is smaller than $60 \mathrm{deg}$ and the distance is shorter than $1 \mathrm{~m}$. (3) The lateral error is negligibly small if the distance to detect is shorter than $1 \mathrm{~m}$. Moreover it shows the result of the examination to apply the range scanner in the Step-On Interface (SOI), in which the scanner is used for detection and measurement of the stepping of an operator. Based on the measured results, we designed the judgment method of the stepping, the installation position of the scanner, and the placement of buttons in the direction screen to apply the range scanner to the SOI for operation of a mobile robot.
\end{abstract}

Keywords: Range scanner, Characteristics measurement, Step-On Interface (SOI), Mobile robot.

\section{INTRODUCTION}

This paper shows the result of the characteristics measurement of a two-dimensional active range scanner, URG made by Hokuyo Automatic Co., Ltd. [1] which was released in 2004 and is spreading widely as an external sensor for mobile robot.

A sensor is indispensable so that a mobile robot can operate robustly in an environment [2]. There are various types of sensor to acquire some information of the external world. The "range sensor" is a general term of a sensor to measure the distance to the surface of an object. The "range scanner" is regarded as a two-dimensional sensor doing planar scanning or a three-dimensional sensor measuring threedimensional object or environment. The range scanner is the same meaning as the range finder [3]. The range scanner measuring the distance to some object in multi-dimensional field is very useful for the environmental recognition and the contact / collision avoidance with building an environmental map and / or estimating the self-location. There are various methods to measure the distance in space out of touch. One of the commonly used methods is the active optical type in which light beam is radiated by itself and the distance to some object is measured. This method has several advantages. It can measure a wide range in a short time when the optical beam scans fast. And it can measure any object less affected by some ambient light.

*Address correspondence to this author at the Bio-Robotics and HumanMechatronics Lab., Shizuoka Univ., Johoku, Naka, Hamamatsu 432-8561, Japan; E-mail: ttmatum@ipc.shizuoka.ac.jp
The product by Sick AG is well known as the active range scanner using laser beam, like LMS-200 [4]. It adopts the TOF (time-of-flight, spread time of light) method to measure in which the distance is calculated from the time between the radiation and the receiving of laser beam reflected on some object. It is difficult to measure the roundtrip time directly because the laser beam advances fast in comparison with the supersonic waves. However this product realizes the high efficiency as $15 \mathrm{~mm}$ of detection precision at a distance up to $80 \mathrm{~m}$. Therefore this product has contributed greatly on progress of the environmental recognition for mobile robot and the study of SLAM (simultaneous localization and mapping) [5]. There are several reports using LMS [6-12]. However it is said that this product is hard to adjust to small mobile robot due to size, weight, consumption electricity, and cost.

URG made by Hokuyo Automatic Co., Ltd. was developed as a small and power-saving sensor to be used on a small service robot. It radiates an amplitude modulated (at about $50 \mathrm{MHz}$ ) laser beam (near-infrared laser of $785 \mathrm{~nm}$ in wavelength). And it detects the reflected light from some object in the radiated direction. Then it calculates the distance based on the phase difference between the radiated light and the reflected light. It projects two kinds of laser beam with different modulation frequency in turn, because it cannot detect the distance longer than two phase differences when the modulation frequency is only one kind. In addition, the laser beam is burst-emitted in a short time to secure the output power of the laser while satisfying the safety class one [13]. This product realizes $4 \mathrm{~m}$ in the maximum detection distance and $10 \mathrm{~mm}$ in the measurement precision, exercising these ingenuities. 
Most of the conventional studies assume that the sensory data provided from a range scanner includes some amount of error while arithmetic processing to build environmental map or to estimate self-location. However when we want to use the sensory data to control the movement of the robot one after another with comparatively high precision, basic information about the error on the measurement is insufficient. In other words, manufactures only provide the basic characteristics, such as measurement range, resolution performance, error-threshold, and so on. They rarely clarify both the margin of error depending on the measurement condition and the frequency to output some error code when the sensor judges incapable measurement. Consequently some robotic researcher who uses a product reports a measurement result in each case at present. For example, the data about LMS200 is reported in a study aiming at the obstacle avoidance of a mobile robot [14]. The data about PBS (range scanner of TOF method using a LED as light source) [15] made by Hokuyo Automatic Co., Ltd. is reported in a study aiming at the environmental recognition in a walking support robot [16].

The principal specifications of URG-X002S (Fig. 1) that Hokuyo Automatic Co., Ltd., provides are shown in Table $\mathbf{1 .}$ It is indicated that the target object is a white paper in 70$\mathrm{mm}$-square. The report about the measurement error of this product shows the following items [17].

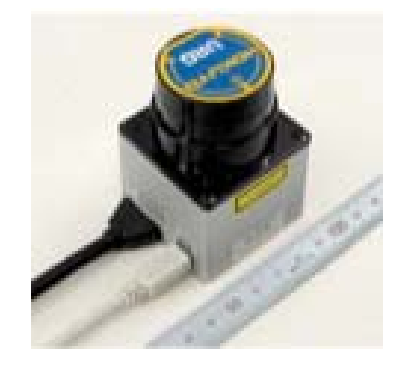

Fig. (1). URG-X002S [1].

Table 1. Specifications of URG-X002

\begin{tabular}{|c|c|}
\hline Item & Specification \\
\hline \hline Light source & Infrared laser (785 nm) \\
\hline Power source & DC 5 V $\pm 5 \%$ \\
\hline Power consumption & less than $5 \mathrm{~mA}$ \\
\hline Detection range & 20 to $4095 \mathrm{~mm}$ \\
\hline Accuracy & $\pm 10 \mathrm{~mm}(20$ to $1000 \mathrm{~mm})$ \\
& $\pm 2 \%$ of distance (1000 to $4095 \mathrm{~mm})$ \\
\hline Angle bounds & 240 deg (step number: 44 to 725$)$ \\
\hline Angular resolution & Step angle: 0.36 deg (360 deg/1024) \\
\hline Scanning time & $100 \mathrm{~ms} / \mathrm{scan}$ \\
\hline Interface & RS-232C, USB Ver. 1.1 \\
\hline Dimensions & 42 to $50 \mathrm{~mm}$ in diameter by $70 \mathrm{~mm}$ in height \\
\hline Weight & $170 \mathrm{~g}$ \\
\hline
\end{tabular}

(1) The wave pattern of the reflected light, in the case that there is no object and the case that the white paper is placed at the distance of $0.1 \mathrm{~m}, 2.0 \mathrm{~m}$, and $4.0 \mathrm{~m}$ respectively.

(2) The measurement limitation distance that is estimated from the light intensity of the reflected light, when the object is various (white paper, black paper, fabric, wooden board, and metal plate).

(3) The average and the standard deviation of the distance value in the case that various objects are located at various distances $(0.2 \mathrm{~m}, 1.0 \mathrm{~m}, 2.0 \mathrm{~m}, 3.0 \mathrm{~m}$, and 4.0 $\mathrm{m})$.

However these data are insufficient to examine whether the distance value provided from the sensor can be used for motion control of robot. In other words, it lacks the data about the margin of error included in measured value and the error rate (the frequency that the scanner judges to be impossible to measure and the error code is output) of various objects, at various directions of object (direction angle, the direction in which some object locates to the scanner) and at various orientations of object (oblique angle, the angle of object surface to the line between the scanner and the object). The production model to improve the processing capabilities by replacing the microcomputer has been commercially supplied [18] and a long-distance type has also been developed [19] after the prototype URG-X002S taken up here. However the data in this paper would be valuable because it is not widely changed both on the measurement principle and the internal constitutions.

The rest of this paper is constructed as follows. Chapter 2 shows the measured result of the distance accuracy and the lateral precision of URG-X002S in various conditions. A part of the measured result has been presented in a domestic meeting [20]. Chapter 3 introduces the Step-On Interface (SOI), in which the scanner is used to detect and measure the stepping of an operator. The concept of the SOI has also been proposed in a domestic meeting [21]. Then in Chapter 4 the measurement experiment of footwear by URG-X002S to realize the SOI is presented. Finally Chapter 5 explains the result of the examination to apply the range scanner to SOI for operation of a mobile robot. We designed the judgment method of the stepping, the installation position of the scanner, and the placement of buttons in the direction screen based on the measured results. The details of the prototype mobile robot that carries the SOI equipment will be presented in following papers.

\section{CHARACTERISTICS MEASUREMENT OF RANGE SCANNER}

We measured the characteristic about the distance accuracy and the lateral precision of URG-X002.

\subsection{Measurement-1 - Distance Accuracy \\ Measurement Purpose}

It is common on a range sensor that the error included in distance value becomes larger and the error rate comes up when the measurement distance becomes longer. Therefore we investigate the accuracy of the distance value depending on the kind of object (material and color), the distance from 
the sensor to the object, the direction of the object to the sensor (direction angle), and the orientation of the object to the sensor (oblique angle).

\section{Measurement Condition}

- $\quad$ Size of object: 70-mm-square (the same size as in the specification list).

- $\quad$ Kind of object: Four kinds - black drawing paper (black surface), white drawing paper (white surface), white polystyrene foam (rough surface), and silver aluminum foil (gloss surface).

- $\quad$ Distance to object: Six kinds - $0.2 \mathrm{~m}, 0.5 \mathrm{~m}, 1.0 \mathrm{~m}, 2.0$ $\mathrm{m}, 3.0 \mathrm{~m}$, and $4.0 \mathrm{~m}$. Those were set corresponding to the range in which the precision was shown in the specification list. The center of the object was put at the distance.

- Direction of object (direction angle): Six kinds - -30 deg, $0 \mathrm{deg}, 30 \mathrm{deg}, 60 \mathrm{deg}, 90 \mathrm{deg}$, and $210 \mathrm{deg}$ assuming the right side of the sensor from the top equal to zero. The distance value at the corresponding step number was recorded when the center of the object put in the direction.

- Orientation of object (oblique angle): Three kinds - 0 $\mathrm{deg}, 30 \mathrm{deg}$, and $60 \mathrm{deg}$.

- Data acquisition: Data (distance value or error code) scanned thirty times in resting state was recorded (Fig. 2).

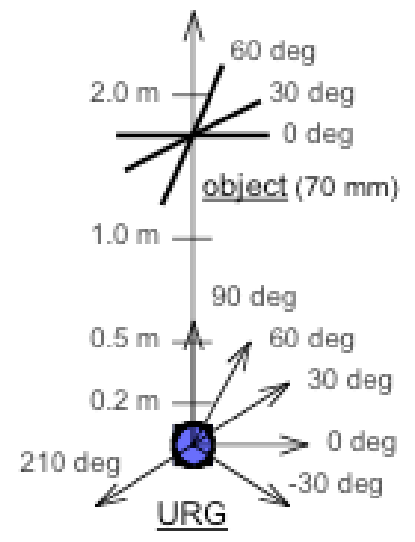

Fig. (2). Experimental condition.

\section{Measurement Result}

Fig. (3) shows the result summarized on the error of the measured distance value depending both on the kind of object and the direction angle (in the case at a distance of 0.2 $\mathrm{m}, 0.5 \mathrm{~m}, 1.0 \mathrm{~m}$, and $2.0 \mathrm{~m}$ ), when the oblique angle and the distance to object were set. The distance value when an error code was not output during thirty times of scanning showed similar tendency. It did not vary widely, and dramatically different values in plus and minus were not provided.

Table 2 shows the error rate and the margin of error (absolute value) between the measured average and the true value about distance, when gathering up the data in every kind of object from all experimental conditions shorter than $1.0 \mathrm{~m}$ (distance: $0.2 \mathrm{~m}, 0.5 \mathrm{~m}$, and $1.0 \mathrm{~m}$ ) which is assumed to be the range to measure when used in SOI (details are explained in the next chapter).

In the case of gloss surface, it generally has a large margin of error. Furthermore the error rate rises when the oblique angle of object becomes large and also the distance to object becomes long (Table 3 ). In the case of black surface, the margin of error is small when the distance value is provided, however an error rate is totally high. Furthermore the error rate rises when the oblique angle of object becomes large and also the distance to object becomes long (Table 4). Tables $\mathbf{3}$ and $\mathbf{4}$ show the error rate of gloss surface and black surface respectively, summarized on the oblique angle and the distance to object regardless of the direction angle of object to the sensor.

In the case of white surface, not only the error rate is zero but also the margin of error is smaller than $20 \mathrm{~mm}$ and the varying is small, if the oblique angle is smaller than $60 \mathrm{deg}$ and the distance is shorter than $1 \mathrm{~m}$. The margin of error is dozens of millimeters, even if the measurement range is up to $4 \mathrm{~m}$ (Table 5). Even in the case of rough surface, if the distance is shorter than $1 \mathrm{~m}$ the error rate is zero and the error is not so variable at around 30 to $40 \mathrm{~mm}$. However when the distance is $4 \mathrm{~m}$, we can get a normal value except in the case that the oblique angle is zero (Table 6). Tables 5 and 6 show the margin of error (absolute value) of white surface and rough surface respectively, summarized on the oblique angle and the distance to object regardless of the direction angle of object to the sensor.

\subsection{Measurement-2 - Lateral Precision}

\section{Measurement Purpose}

The position information in the right and left direction (lateral direction) from the sensor, as well as the distance information in the front and back direction (longitudinal direction), is necessary to identify the step-on position of an operator in SOI (details are explained in the next chapter). Therefore the reliability of data in lateral direction is investigated.

\section{Measurement Condition}

- $\quad$ Size of object: 70-mm-square (the same size as in the measurement-1).

Table 2. Experimental Result (Distance: $0.2 \mathrm{~m}, 0.5 \mathrm{~m}, 1.0 \mathrm{~m}$ )

\begin{tabular}{|c|c|c|c|c|}
\hline Test Object & Black & White & Rough & Gloss \\
\hline \hline $\begin{array}{c}\text { Margin of error mm Average } \\
\text { (Standard deviation) }\end{array}$ & $\begin{array}{c}12.3 \\
(8.4)\end{array}$ & $\begin{array}{c}14.9 \\
(3.8)\end{array}$ & $\begin{array}{c}60.9 \\
(4.5)\end{array}$ \\
\hline Error rate \% & 32.7 & 0 & 0 & 25.1 \\
\hline
\end{tabular}



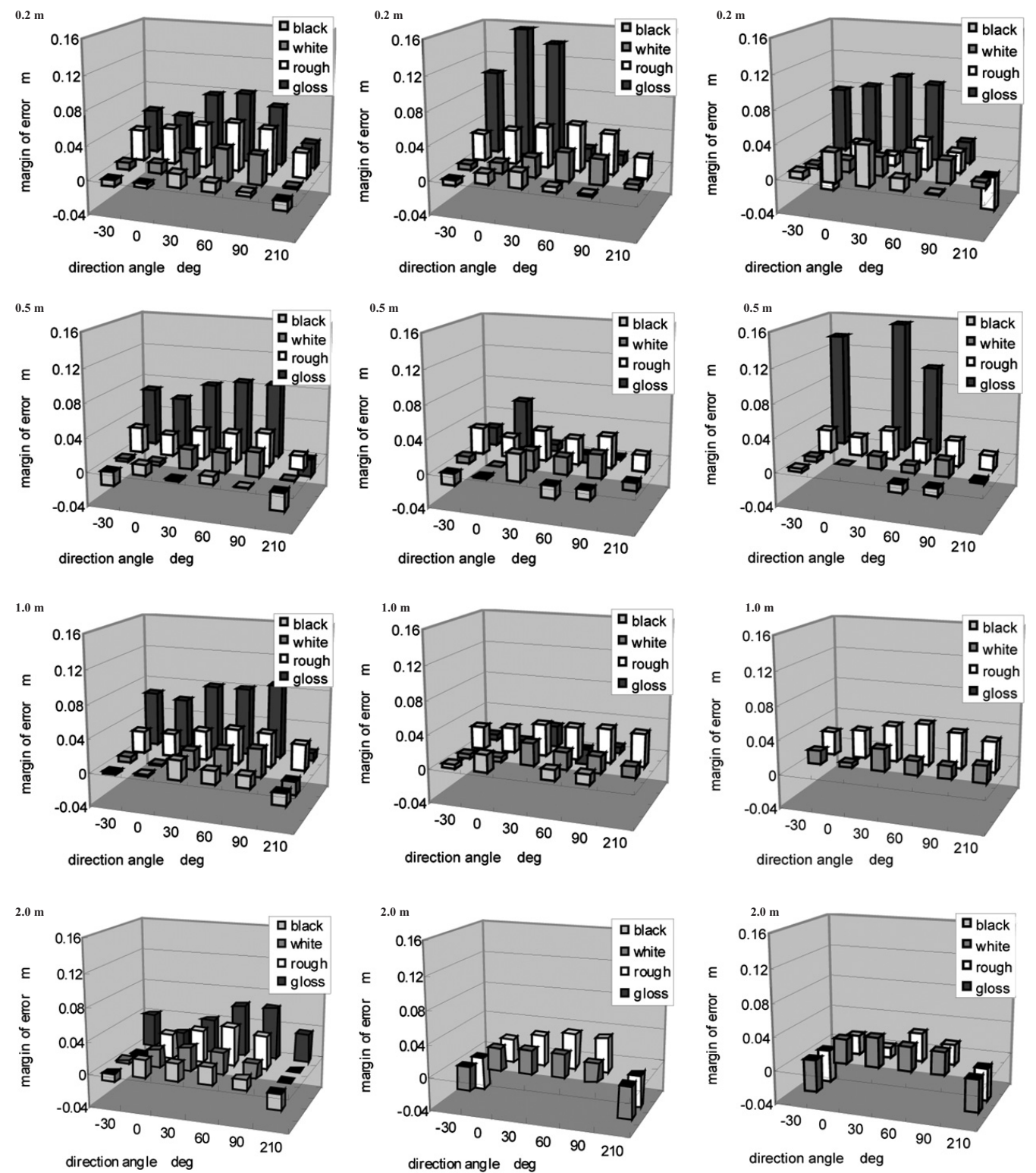

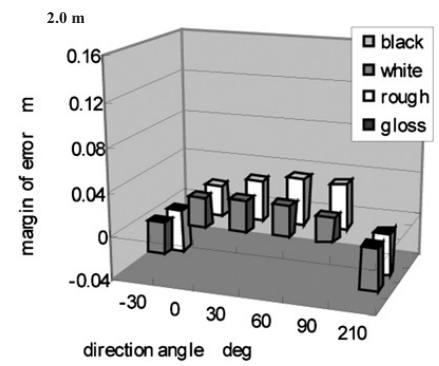

(b) Oblique angle: $30 \mathrm{deg}$

(a) Oblique angle: $0 \mathrm{deg}$

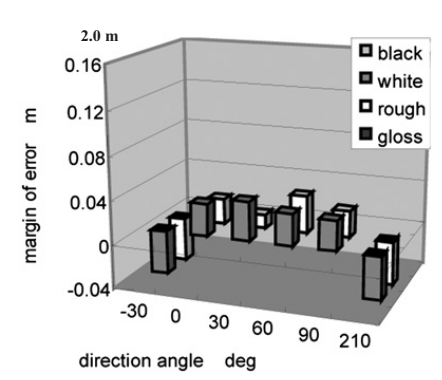

(c) oblique angle: $60 \mathrm{deg}$

Fig. (3). Experimental result (distance: $0.2 \mathrm{~m}, 0.5 \mathrm{~m}, 1.0 \mathrm{~m}$, and $2.0 \mathrm{~m}$ ).

Table 3. Error Rate of Gloss Surface

\begin{tabular}{|c|c|c|c|}
\hline \multirow{2}{*}{ Distance } & \multicolumn{3}{|c|}{ Oblique Angle } \\
\cline { 2 - 4 } & $\mathbf{0 ~ d e g}$ & $\mathbf{3 0} \mathbf{~ d e g}$ & $\mathbf{6 0 ~ d e g}$ \\
\hline \hline $0.2 \mathrm{~m}$ & 0.0 & 16.7 & 16.7 \\
\hline $0.5 \mathrm{~m}$ & 0.0 & 16.7 & 59.2 \\
\hline $1.0 \mathrm{~m}$ & 0.0 & 16.7 & 100.0 \\
\hline $2.0 \mathrm{~m}$ & 0.0 & 100.0 & 100.0 \\
\hline $3.0 \mathrm{~m}$ & 0.0 & 100.0 & 100.0 \\
\hline $4.0 \mathrm{~m}$ & 0.0 & 100.0 & 100.0 \\
\hline
\end{tabular}

unit: \%

- Kind of object: Four kinds (the same object as in the measurement-1).
- Distance to object and direction of object (direction angle): Supposing that the far side of the projected screen is at the distance of $60 \mathrm{~cm}$ from the sensor in SOI, when an object is put around the corner of the screen of $73 \mathrm{~cm}$ in width, the right rear, the front rear, and the left rear on the screen are corresponding to the direction angle of $60 \mathrm{deg}, 90 \mathrm{deg}$, and $120 \mathrm{deg}$ from the sensor respectively. We acquired the distance value at the step number corresponding to those directions.

- Orientation of object (oblique angle): $0 \mathrm{deg}$ facing to the sensor.

- Data acquisition: Data (distance value or error code) scanned ten times in resting state was recorded.

\section{Measurement Result}

Table 7 shows the number of steps and the bounds of step number as the measured value and the theoretical value at 
Table 4. Error Rate of Black Surface

\begin{tabular}{|c|c|c|c|}
\hline \multirow{2}{*}{ Distance } & \multicolumn{3}{|c|}{ Oblique Angle } \\
\cline { 2 - 4 } & $\mathbf{0 ~ d e g}$ & $\mathbf{3 0} \mathbf{~ d e g}$ & $\mathbf{6 0} \mathbf{~ d e g}$ \\
\hline \hline $0.2 \mathrm{~m}$ & 0.0 & 20.7 & 33.8 \\
\hline $0.5 \mathrm{~m}$ & 0.0 & 22.3 & 63.0 \\
\hline $1.0 \mathrm{~m}$ & 0.0 & 54.2 & 100.0 \\
\hline $2.0 \mathrm{~m}$ & 15.0 & 100.0 & 100.0 \\
\hline $3.0 \mathrm{~m}$ & 100.0 & 100.0 & 100.0 \\
\hline $4.0 \mathrm{~m}$ & 100.0 & 100.0 & 100.0 \\
\hline
\end{tabular}

unit: $\%$

Table 5. Margin of Error of White Surface

\begin{tabular}{|c|c|c|c|}
\hline \multirow{2}{*}{ Distance } & \multicolumn{3}{|c|}{ Oblique Angle } \\
\cline { 2 - 4 } & $\mathbf{0 ~ d e g}$ & $\mathbf{3 0} \mathbf{~ d e g}$ & $\mathbf{6 0 ~ d e g}$ \\
\hline \hline $0.2 \mathrm{~m}$ & 9.2 & 18.8 & 17.9 \\
\hline $0.5 \mathrm{~m}$ & 18.2 & 15.1 & 8.9 \\
\hline $1.0 \mathrm{~m}$ & 14.3 & 15.3 & 16.9 \\
\hline $2.0 \mathrm{~m}$ & 16.7 & 33.3 & 41.9 \\
\hline $3.0 \mathrm{~m}$ & 29.3 & 30.7 & 42.1 \\
\hline $4.0 \mathrm{~m}$ & 54.4 & 69.9 & 74.5 \\
\hline
\end{tabular}

unit: $\mathrm{mm}$

Table 6. Margin of Error of Rough Surface

\begin{tabular}{|c|c|c|c|}
\hline \multirow{2}{*}{ Distance } & \multicolumn{3}{|c|}{ Oblique Angle } \\
\cline { 2 - 4 } & $\mathbf{0 ~ d e g}$ & $\mathbf{3 0} \mathbf{~ d e g}$ & $\mathbf{6 0} \mathbf{~ d e g}$ \\
\hline \hline $0.2 \mathrm{~m}$ & 44.7 & 41.6 & 38.7 \\
\hline $0.5 \mathrm{~m}$ & 30.0 & 30.1 & 26.1 \\
\hline $1.0 \mathrm{~m}$ & 34.0 & 35.7 & 38.3 \\
\hline $2.0 \mathrm{~m}$ & 26.1 & 44.2 & 41.2 \\
\hline $3.0 \mathrm{~m}$ & 44.8 & 46.1 & 62.9 \\
\hline $4.0 \mathrm{~m}$ & 67.7 & (no) & (no) \\
\hline
\end{tabular}

unit: $\mathrm{mm}$

which the distance value in an appropriate range was acquired or should be obtained because the object was $70 \mathrm{~mm}$ in width. This measurement result is concluded as follows.

- $\quad$ The kind (color and material) of object rarely affects on the lateral precision.

- The precision is high so that the lateral error is within a step $(0.36 \mathrm{deg})$ of the scanner.

It is thought that when an object is far from the sensor further the lateral error will increase as the distance error. However it was found that the lateral error was negligibly small if the distance to detect was shorter than $1 \mathrm{~m}$.
Table 7. Lateral Error Measurement of Test Object

\begin{tabular}{|c|c|c|c|}
\hline \multirow{2}{*}{ Distance } & \multicolumn{3}{|c|}{ Direction Angle } \\
\cline { 2 - 4 } & $\mathbf{6 0} \mathbf{~ d e g}$ & $\mathbf{9 0 ~ d e g}$ & $\mathbf{1 2 0} \mathbf{~ d e g}$ \\
\hline \hline black surface & $\begin{array}{c}18 \\
(267-284)\end{array}$ & $\begin{array}{c}17 \\
(334-350)\end{array}$ & $\begin{array}{c}17 \\
(399-415)\end{array}$ \\
\hline white surface & 18 & 17 & 16 \\
$(267-284)$ & $(333-349)$ & $(400-415)$ \\
\hline rough surface & 17 & 17 & 16 \\
gloss surface & $\begin{array}{c}17 \\
(267-283)\end{array}$ & $\begin{array}{c}18 \\
(334-350)\end{array}$ & $\begin{array}{c}18 \\
(333-350)\end{array}$ \\
\hline ideal value & $\begin{array}{c}17 \\
(267-283)\end{array}$ & $\begin{array}{c}17 \\
(333-349)\end{array}$ & $\begin{array}{c}16 \\
(399-414)\end{array}$ \\
\hline
\end{tabular}

unit: number of steps (step number).

\section{REALIZATION OF STEP-ON INTERFACE USING RANGE SCANNER}

\subsection{Step-On Interface}

The Step-On Interface (SOI) is a new interface to direct some task and operation to robotic or mechartonic system, in which a projector projects and displays a direction screen on a running surface or a floor face, and an operator specify the button which shows the desired contents to order by stepping or pointing. The SOI would be equipped, for example, on a robot which supports duties in hospital or nursing facility (Fig. 4) [22]. There are many cases that still more supports by additional hands are required while medical assistants or

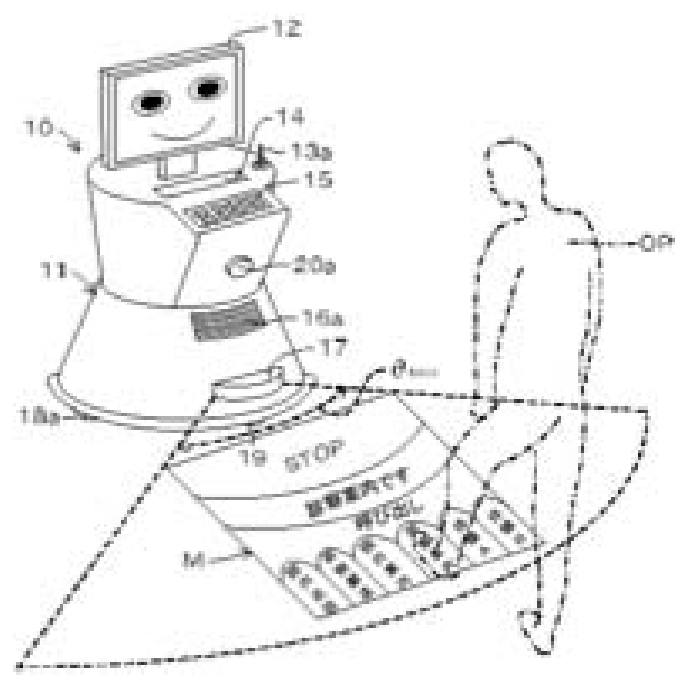

Fig. (4). SOI on in-hospital service support robot [22].

care workers use their both hands to support the medical service by doctor, the nursing to patients, the caring for elderly, and so on while chronic labor shortage in dropping birthrate and aging society. Accordingly a human-coexisting type robot which performs, for example, delivery or conveyance of medical appliances, patient's records, meals, and so on is desired. The SOI might be used on such kind of hu- 
man-coexisting type robot which operates nearby people and supports tasks for people. On the other hand, a user who doesn't have any remote-control unit can operate and direct a robot without approaching the robot when the direction screen is projected around the user from the robot. It benefits for people who is anxious about what a robot will do next and do not want to approach it. The SOI has a novelty that a screen projected from a projector is used as a bilateral interface. It not only presents the information from the machine to a person but also assigns the instruction from a person to the machine. The SOI has the following features.

(1) The SOI can be used in hands-free contrary to keyboard, mouse, touch panel, or joystick. Therefore senior, handicapped, and injured people who are inconvenient to move their hands and fingers and also people who are busy working on his hands may direct a robotic or mechatronic system.

(2) An operator can direct without disturbing neighboring quietness and even under noisy environment because the SOI is not voice input.

(3) Any special device is unnecessary and an operator can use his own foot or walking stick usually used, although the eye control requires some special devices to detect eye movement and it imposes various constraints such as the position and orientation where an operator can direct.

(4) An operator only moves his foot or stick roughly to direct instead of accurate and precise movement of hands or fingers.

(5) Anywhere may become a direction screen without preliminary preparation or special setting on the environment because the direction screen is projected.

(6) Even a beginner or a foreigner can direct immediately even if details are not explained preliminary because the contents of movement or the task to direct can be displayed not only by letters but also by icons (figures and pictures).

(7) It is easy to set and modify the function and the placement in the direction screen because it is written in a software program.

\subsection{SOI Using Range Scanner}

The mobile robot HFAMRO-1 (Human-Friendly Amusing Mobile RObot -1) was developed which carries the SOI equipment consisting of a projector and a range scanner on an omni-directional mobile mechanism. The range scanner detects and measures the stepping of an operator to identify the operation button directed. In HFAMRO-1, the size of the projected screen becomes 36 -inch type $(550 \mathrm{~mm}$ in height by $730 \mathrm{~mm}$ in width) while making the projection distance the shortest $(1.2 \mathrm{~m})$ of the adopted product to let the size of the robot with the SOI equipment small as much as possible. The procedure in which an operator directs the movement of the robot is as follows (Fig. 5).

(1) The projector projects a direction screen on a running surface.

(2) An operator steps on an appropriate place in the direction screen with his foot.

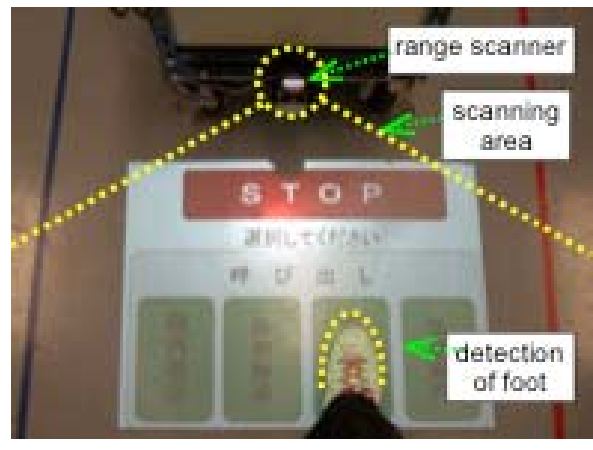

Fig. (5). Direction of robot movement.

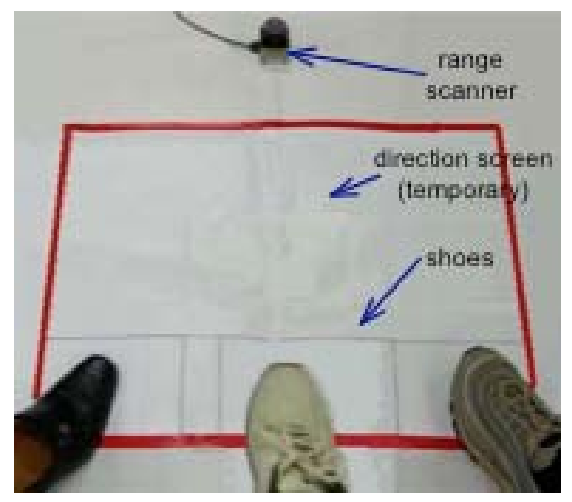

Fig. (6). Lateral error measurement of worn shoes.

Table 8. Lateral Error Measurement of Worn Shoes

\begin{tabular}{|c|c|c|c|c|}
\hline \multirow{2}{*}{ Distance } & \multicolumn{4}{|c|}{ Direction Angle } \\
\cline { 2 - 5 } & $\mathbf{6 0} \mathbf{~ d e g}$ & $\mathbf{9 0} \mathbf{~ d e g}$ & $\mathbf{1 2 0} \mathbf{~ d e g}$ & ideal Value \\
\hline \hline black gloss & $\begin{array}{c}2 \\
(273-274)\end{array}$ & $\begin{array}{c}1 \\
(342)\end{array}$ & $\begin{array}{c}0 \\
(\mathrm{no})\end{array}$ & $15-1$ \\
\hline white matte & $\begin{array}{c}22 \\
(265-286)\end{array}$ & $\begin{array}{c}24 \\
(335-358)\end{array}$ & $\begin{array}{c}22 \\
(402-423)\end{array}$ & $22-24$ \\
\hline gray clothe & $\begin{array}{c}18 \\
(264-281)\end{array}$ & $\begin{array}{c}19 \\
(336-354)\end{array}$ & $\begin{array}{c}18 \\
(403-420)\end{array}$ & $18-19$ \\
\hline
\end{tabular}

unit: number of steps (step number).

(3) The range scanner detects the stepping and measures the position of stepping.

(4) The robot performs the content of the directed operation button.

\section{MEASUREMENT EXPERIMENT OF FOOTWEAR BY RANGE SCANNER}

The measurement experiment of footwear by URG-X002 was carried out assuming the situation that an operator stepped on an operation button in the direction screen projected on a running surface.

\subsection{Pilot Study using Worn Shoes}

As a trial we measured the laterals precision using three kinds of worn shoes - enameled-black leather, matte-white leather, and gray fabric - like the previous chapter. The measurement situation is illustrated in Fig. (6). Table 8 
shows the number of steps and the bounds of step number as the measured value and the theoretical value at which the distance value in an appropriate range was acquired. It was almost impossible to acquire the distance value in appropriate range on the shoes with enameled-black leather. This might be because the black color absorbs the light from the light source of the scanner. Furthermore most of the light reflected in the specific direction by the shiny surface. And then there was few amount of reflected light back to the scanner. On other shoes, some appropriate distance value was acquired at the step number calculated from the width of shoes.

Considering the result of this pilot study with the result of the characteristics measurement, it would appear that the range scanner could detect and measure the stepping with the commonly used shoes except black and highly-grazed.

\subsection{Basic Experiment Using Test Footwear}

It may be difficult on the SOI equipment using a range scanner to completely deal with all shoes which a person wears considering the result of the previous section. Therefore we prepared a standard footwear for test and carried out the basic experiment using the test footwear.

We prepared a light-green slipper with a yellow decoration ball in the tip as the footwear which would be easy for everyone to wear and remove (Fig. 7). The diameter of the decoration ball is $50 \mathrm{~mm}$, and the total size of the footwear is $90 \mathrm{~mm}$ in height, $100 \mathrm{~mm}$ in width, and $265 \mathrm{~mm}$ in length. The operation button on the projected screen was decided to 50-mm-square according to the size of the decoration ball. An operator will step on the operation button with his tiptoe being aware of his thenar eminence.

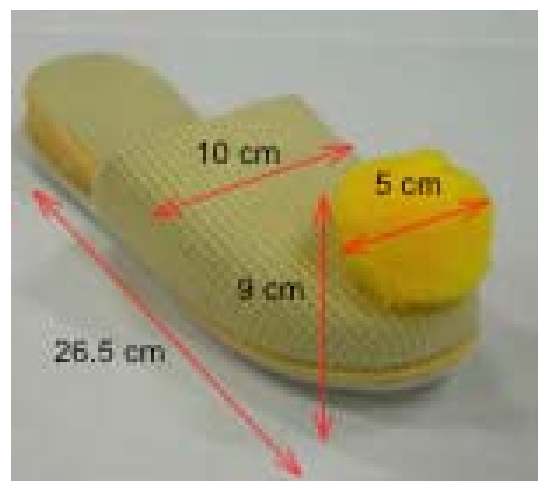

Fig. (7). Test footwear.

\subsubsection{Experiment-1 - Influence of Projected Light}

\section{Experiment Objective}

The position of stepping by an operator in the projected screen must be detected precisely in the SOI. Therefore we have to confirm whether the light projected from the projector influence the data acquired with the range scanner.

\section{Experimental Condition}

- $\quad$ Light condition: Five kinds - light from the projector (white, blue, and black which is designated in RGB), fluorescent lamp, and darkness.

- Object: Test footwear, in front of the scanner, at the position of $60 \mathrm{~cm}$ to the tip, facing to the scanner at the oblique angle of $0 \mathrm{deg}$.

- Date acquisition: Data (distance value or error code) scanned ten times in resting state was recorded.

\section{Experimental Result}

Table 9 shows the number of steps and the bounds of the step number, at which the appropriate distance value was acquired, and also the average of the distance value acquired at the step number corresponding to the front of the scanner. From the result, it is confirmed that the projected light rarely affects the distance value and the number of steps that appropriate distance value is acquired so as to be negligible.

Table 9. Influence of Projected Light (Footwear)

\begin{tabular}{|c|c|c|}
\hline Lighting Condition & $\begin{array}{c}\text { Num. of Steps } \\
\text { (Step Number) }\end{array}$ & Distance cm \\
\hline \hline white light (projector) & $13(334-347)$ & 59.12 \\
\hline blue light (projector) & $13(334-347)$ & 59.12 \\
\hline black light (projector) & $13(334-347)$ & 59.15 \\
\hline fluorescent lighting & $14(334-348)$ & 58.97 \\
\hline darkness & $13(334-347)$ & 59.09 \\
\hline ideal value & 14 & 60.0 \\
\hline
\end{tabular}

\subsubsection{Experiment-2 - Detection of Stepping Position}

\section{Experiment Objective}

The objective is to declare the detection precision when a button is stepped on in the direction screen.

\section{Experimental Condition}

- $\quad$ Placement of buttons: 50-mm-square operation buttons are arranged in three places in the rectangular frame assumed as a projected screen.

$>$ Button-1 - At the distance of $67.5 \mathrm{~cm}$ from the scanner to the center of the button in the direction angle of $60 \mathrm{deg}$.

$>$ Button-2 - At the distance of $62.5 \mathrm{~cm}$ from the scanner to the center of the button in the direction angle of 90 deg (front).

$>$ Button-3 - At the distance of $67.5 \mathrm{~cm}$ from the scanner to the center of the button in the direction angle of $120 \mathrm{deg}$.

- Object: Test footwear, putting on an operation button so that the center of the decoration ball is on the center of the button, at three kinds of oblique angle, $-45 \mathrm{deg}, 0$ $\mathrm{deg}$, and $45 \mathrm{deg}$.

- Data acquisition: Data (distance value or error code) scanned ten times in resting state was recorded.

\section{Experimental Result}

Table 10 shows the average of the distance value acquired at the step number passing through the center of the button with the ideal value. From these results, it is found 
that the error between the average and the ideal value about the distance is around $20 \mathrm{~mm}$ at the maximum.

Table 10. Average of Measured Distance (footwear)

\begin{tabular}{|c|c|c|c|}
\hline \multirow{2}{*}{ Direction Angle Deg } & \multicolumn{3}{|c|}{ Distance cm } \\
\cline { 2 - 4 } & Button-1 & Button-2 & Button-3 \\
\hline \hline-45 & 65.7 & 61.6 & 67.2 \\
\hline 0 & 65.6 & 60.0 & 67.1 \\
\hline 45 & 67.1 & 60.9 & 66,2 \\
\hline ideal value & 65.0 & 60.0 & 65.0 \\
\hline
\end{tabular}

The bounds of the step number in each button in which the distance value to the footwear was acquired is shown in Fig. (8). In each case, the step number (horizontal short-line in the figure) passing through the center of the button is in the bounds (vertical line in the figure) of the step number that the distance value was acquired. In addition, from the measurement result when making the oblique angle of the footwear different, it is confirmed that appropriate distance
In addition, the range of distance from the sensor to the center of the button plus or minus $50 \mathrm{~mm}$ is made into the scope of detection for the distance value. It would be judged that an operator has stepped on the operation button to direct the contents of the button, when the distance value at all five steps is within the scope. This is based on the size of button (50-mm-square) and the measurement error (around $20 \mathrm{~mm}$ at the maximum) of the distance value, considering individual difference of operators about the relative position between the button and the foot or footwear.

\subsection{Installation Position of Scanner}

It is decided to install the scanner upside down at the front-and-center of the main body in HFAMRO-1 so that the sensing part is at the height of $40 \mathrm{~mm}$ from a running surface. Even if there is some unevenness on a running surface, it might not touch the scanner because there is a gap more than $10 \mathrm{~mm}$ between the lower part of the scanner and the running surface.

The horizontal relationship between the range scanner and the direction screen $(550 \mathrm{~mm}$ in height by $730 \mathrm{~mm}$ in width) is shown in Fig. (9). The range scanner scans over the

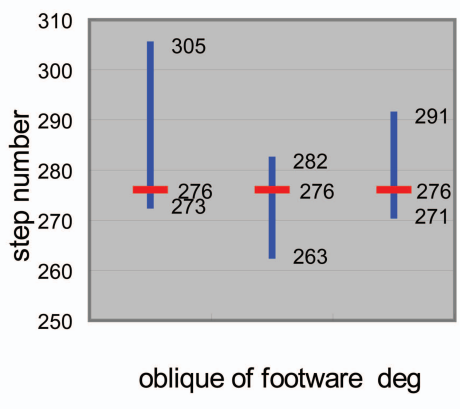

(a) Button-1

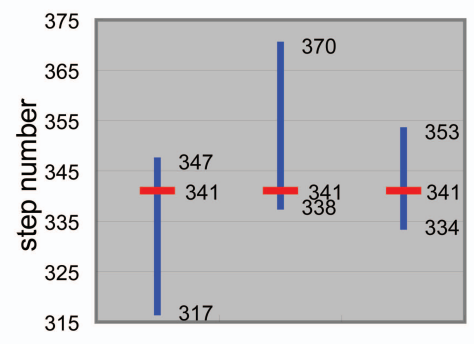

oblique of footware deg

(b) Button-2

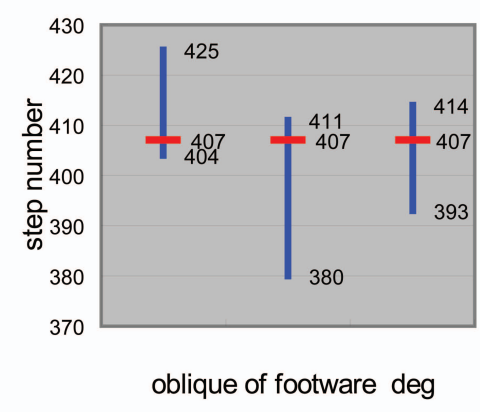

(c) Button-3

Fig. (8). Bounds of step number in which distance value is acquirable.

value can be acquired at more than three steps on both sides from the step passing through the center of the button.

\section{DESIGN TO APPLY RANGE SCANNER TO SOI}

Based on the result both of the measurement in Chapter 2 and the experiment in Chapter 4, we designed the judgment method of stepping, the installation position of the scanner, and the placement of buttons in the direction screen to apply the range scanner to SOI for operation of a mobile robot.

\subsection{Judgment Method of Stepping}

It is decided that the existence of foot or footwear on a certain operation button is judged using the distance value at five steps totally, including each two steps right and left with the step passing through the center of the button. This is based on the result of the basic experiment using test footwear. There is a risk of misjudgment due to measurement errors when checking the distance value only at the step number passing through the center of the button. The five steps are equivalent to the direction angle of $1.4 \mathrm{deg}$, for example, which is equivalent to $21 \mathrm{~mm}$ in right and left at the distance of $86 \mathrm{~cm}$ from the scanner.

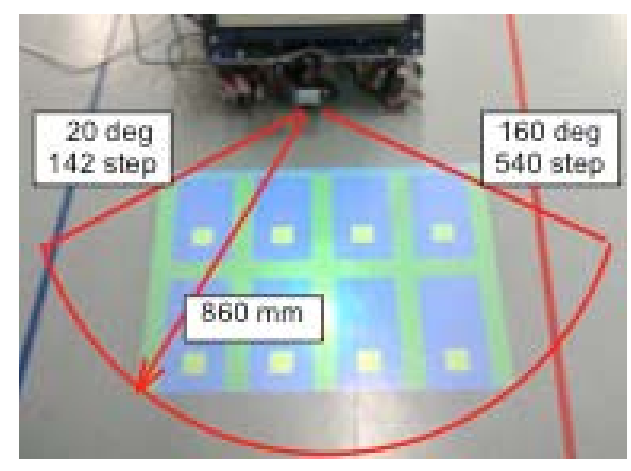

Fig. (9). Positional relationship between direction screen and range scanner.

direction screen in the range of 20 to 160 deg (step number: 142 to 540) and at the distance of 20 to $860 \mathrm{~mm}$.

\subsection{Placement of Buttons in Direction Screen}

It is decided that eight buttons of 50-mm-square are arranged at two lines by four sequences in the direction screen (550 $\mathrm{mm}$ in height by $730 \mathrm{~mm}$ in width). Two lines in front- 
back direction is decided referencing the report about the full length of foot $(21.1$ to $28.7 \mathrm{~cm})$ [23] and the most common size of footwear (25EE) of male adults (18 to 60 years old) in Japan. The buttons can be arranged by five sequences when securing the distance between buttons at about the width of shoes in order to make sure not to step on the neighborhood button at the same time. However, four sequences in lateral direction is decided considering the case that an operator steps on not standing in front of the direction screen strictly or he steps with twisting his ankle. The designed placement of buttons in the direction screen is illustrated in Fig. (10).

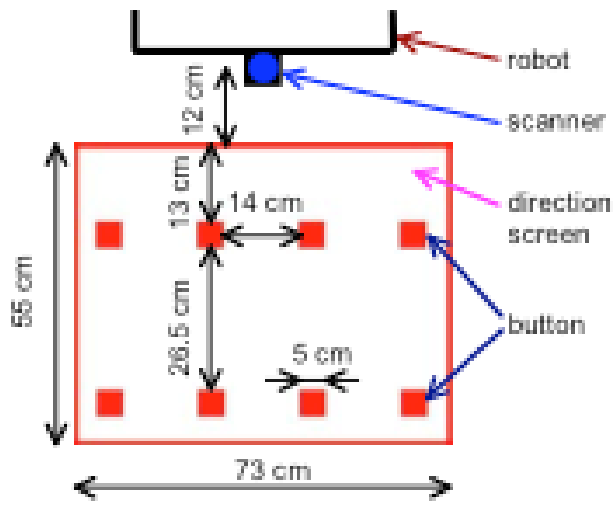

Fig. (10). Placement of buttons in direction screen.

\section{CONCLUSION}

This paper showed the result of the characteristics measurement of a two-dimensional active range scanner, URG made by Hokuyo Automatic Co., Ltd. which was released in 2004 and is spreading widely as an external sensor for mobile robot. Specifically it presented the measured result of the distance accuracy and the laterals precision of the prototype URG-X002S in various conditions. Moreover it showed the result of the examination to apply the range scanner in the Step-On Interface (SOI), in which the scanner is used for detection and measurement of the stepping of an operator. Based on the measured results, we designed the judgment method of the stepping, the installation position of the scanner, and the placement of buttons in the direction screen to apply the range scanner to the SOI for operation of a mobile robot.

The following items were clarified from the characteristics measurement of URG-X002S.

(1) In the case that the object has a gloss surface or a black surface, the error rate (the frequency that the scanner judges to be impossible to measure and an error code is output) rises when the oblique angle of object becomes large and also the distance to object becomes long.

(2) In the case that the object has a white surface or a rough surface, not only the error rate is zero but also the margin of error becomes dozens of millimeters and the varying is small, if the oblique angle is smaller than 60 deg and the distance is shorter than $1 \mathrm{~m}$.

(3) The lateral error is negligibly small if the distance to detect is shorter than $1 \mathrm{~m}$.
From the measurement experiment of footwear, we found the following.

(1) The range scanner can detect and measure the stepping with the commonly used shoes except black and highlygrazed.

(2) The projected light rarely affects the measurement so as to be negligible.

Based on the result of the measurement and experiment, it was decided that in the SOI the existence of foot or footwear on a certain operation button is judged using the distance value at five steps totally, including each two steps right and left with the step passing through the center of the button. Furthermore, the installation position (sensing part is at the height of $40 \mathrm{~mm}$ from a running surface) of the scanner to the robot body and the placement (eight buttons of 50$\mathrm{mm}$-square at two lines by four sequences) of buttons in the direction screen $(550 \mathrm{~mm}$ in height by $730 \mathrm{~mm}$ in width) were also designed.

This paper introduced the SOI using the range scanner to detect and measure the stepping of an operator as the case study to utilize the result of the characteristics measurement of the range scanner. The result of the characteristics measurement provided here is valuable and applicable, for example, to the movement control based on the measured data for all kinds of robot with the range scanner.

\section{ACKNOWLEDGMENTS}

Gratitude is expressed to laboratory members with selfsacrificing dedications, especially to Mr. Mingtong Lin (now, Fujikiko Co., Ltd.) and Mr. Hiroshi Yamamori (now, Mitsubishi Electric Co.) who greatly contributed on the measurement of scanner and the data reduction, and to $\mathrm{Mr}$. Seigo Honda (now, Hitachi, Ltd.) and Mr. Kosuke Akai (now, graduate student of Shizuoka Univ.) who significantly contributed on the development of prototype mobile robot.

\section{REFERENCES}

[1] Hokuyo Automatic Co., Ltd., "Photo sensor", Hokuyo Automatic Co., Ltd., 2008. [On-line] Available: www.hokuyo-aut.jp/02sensor/ [Accessed Nov. 15, 2008]

[2] A. Ohya, "Environmental Model for Autonomous Movement", in Robotics Society in Japan (ed.), Robotics Handbook, Tokyo: Corona Publishing, 2005, pp. 612-614.

[3] S. Yuta, A. Ohya, and N. Shimaji, "Commercial Production of Optical Range-sensor for Intelligent Robot", Journal of the Robotics Society of Japan, vol. 23, no. 2, pp. 181-184, 2005.

[4] SICK AG, "Auto Ident", SICK AG, 2007. [On-line] Available: www.sick.com/home/factory/catalogues/auto/ [Accessed Nov. 15, 2008].

[5] J. J. Leonard and H. F. Durrant-Whyte, "Simultaneous map building and localization for an autonomous mobile robot", in Proc. IEEE/RSJ Int. Workshop on Intelligent Robots and Systems '91 (IROS '91), Nov. 3-5, 1991, Osaka, Japan. Piscataway, NJ: IEEE, 1991, pp.1442-1447.

[6] K. Nagatani, H. Ishida, S. Yamanaka, and Y. Tanaka, "Threedimensional localization and mapping for mobile robot in disaster environments", in Proc. of 2003 IEEE/RSJ Int. Conf. on Intelligent Robots and Systems (IROS 2003), Oct. 27-31, 2003, Las Vegas, NV, USA. Piscataway, NJ: IEEE, 2003, pp.3112-3117.

[7] M. Montemerlo and S. Thrun, "A multi-resolution pyramid for outdoor robot terrain perception", in Proc. of AAAI 19th National Conf. on Artificial Intelligence (AAAI-04), July 25-29, 2004, San Jose, CL, USA. Menlo Park, CL: AAAI, 2004, pp.464-469.

[8] O. Wulf, K.O. Arras, H.I. Christensen, and B. Wagner, "2D mapping of cluttered indoor environments by means of 3D perception”, 
in Proc. of 2004 IEEE Int. Conf. on Robotics and Automation (ICRA 2004), April 26-May 1, 2004, New Orleans, LA, USA. Piscataway, NJ: IEEE, 2004, pp.4204-4209.

[9] S. Kolski, D. Ferguson, C. Stachniss, and R. Siegwart, "Autonomous driving in dynamic environments", in Proc. Workshop on Safe Navigation in Open and Dynamic Environments at the 2006 IEEE/RSJ Int. Conf. on Intelligent Robots and Systems (IROS 2006), Oct. 9-15, 2006, Beijing, China. Piscataway, NJ: IEEE, 2006.

[10] P. Lamon, C. Stachniss, R. Triebel, P. Pfaff, C. Plagemann, G. Grisetti, S. Kolski, W. Burgard, and R. Siegwart, "Mapping with an Autonomous Car", in Workshop on Safe Navigation in Open and Dynamic Environments at the 2006 IEEE/RSJ Int. Conf. on Intelligent Robots and Systems (IROS 2006), Oct. 9-15, 2006, Beijing, China. Piscataway, NJ: IEEE, 2006.

[11] A. Romeo and L. Montano, "Environment understanding: Robust feature extraction from range sensor data", in Proc. of 2006 IEEE/RSJ Int. Conf. on Intelligent Robots and Systems (IROS 2006), Oct. 9-15, 2006, Beijing, China. Piscataway, NJ: IEEE, 2006, pp.3337-3343.

[12] K. O. Arras, O. M. Mozos, and W. Burgard, "Using boosted features for the detection of people in 2d range data", in Proc. of 2007 IEEE Int. Conf. on Robotics and Automation (ICRA 2007), April 10-14, 2007, Roma, Italy. Piscataway, NJ: IEEE, 2007, pp. 34023407.

[13] IEC 60825-1: "Safety of laser products - Part 1: Equipment classification and requirements", International Electrotechnical Commission, 2007-03-30.

[14] C. Ye and J. Borenstein, "Characterization of a 2-D Laser scanner for mobile robot obstacle negotiation", in Proc. of 2002 IEEE Int. Conf. on Robotics and Automation (ICRA '02), May 11-15, 2002, Washington D.C., USA. Piscataway, NJ: IEEE, 2002, pp. 25122518.

[15] Hokuyo Automatic Co., Ltd., "PBS (Distance measurement type obstacle detector)", Hokuyo Automatic Co., Ltd., 2008. [On-line] Available: www.hokuyo-aut.jp/02sensor/07scanner/pbs.html [Accessed Nov. 15, 2008].
[16] M. Alwan, M. B Wagner, G. Wasson, and P. Sheth, "Characterization of infrared range-Finder PBS-03JN for 2-D mapping", in Proc. of 2005 IEEE Int. Conf. on Robotics and Automation (ICRA 2005), April 18-22, 2005, Barcelona, Spain. Piscataway, NJ: IEEE, 2005, pp. 3936-3941.

[17] H. Kawata, A. Phya, S. Yuta, W. Santosh, and T. Mori, "Development of ultra-small lightweight optical range sensor system", in Proc. of 2005 IEEE/RSJ Int. Conf. on Intelligent Robots and Systems (IROS 2005), August 2-6, 2005, Edmonton, Alberta, Canada. Piscataway, NJ: IEEE, 2005, pp.1078-1083.

[18] N. Shimaji, M. Hino, M. Maeda, H. Izumi, T. Mori, H. Kizukuri, M. Yamashita, I. Murata, H. Kawata, and S. Yuta, "Development of ultra-small lightweight optical range-sensor for mobile robots", Journal of the Robotics Society of Japan, vol. 25, no.1, pp. 76-77, 2007.

[19] H. Kawata, T. Mori, and S. Yuta, "Design and development of SOKUIKI sensor URG series for recognizing the environment by mobile robots", in Robotics and Mechatronics Conference 2005 (ROBOMEC 2005), June 10-11, 2005, Kobe, Japan. Tokyo, Japan: JSME, 2005, 2A1-N-032.

[20] M. Lin, H. Yamamori, S. Honda, T. Matsumaru, "Teleoperation of Human-Friendly Robot (42 $2^{\text {nd }}$ report) -Examination of foot position sensing with a URG sensor-", SICE-Chubu Shizuoka 2006, Dec. 25, 2006, Hamamatsu, Japan. Tokyo, Japan: SICE, 2006, 2 D2.

[21] K. Akai, S. Honda, M. Lin, T. Matsumaru, "Teleoperation of Human-Friendly Robot (43rd report) -Examination of software constitution of HFAMRO-", SICE-Chubu Shizuoka 2006, Dec. 25, 2006, Hamamatsu, Japan. Tokyo, Japan: SICE, 2006, 2 D3.

[22] T. Matsumaru: "Operating Device", Patent Abstracts of Japan, 2008-006551, Jan. 17 2008. [On-Line] Available www.ipdl.inpit.go.jp/homepg_e.ipdl [Accessed Nov. 15, 2008].

[23] DHL-AIST, "Japanese Body Dimension Data, 1997-98", AIST, 2005. [On-Line] Available www.dh.aist.go.jp/database/bodydi$\mathrm{meDB} /[$ Accessed Nov. 15, 2008].

Received: November 25, 2008

Revised: February 13, 2009

Accepted: February 15, 2009

(C) Takafumi Matsumaru; Licensee Bentham Open.

This is an open access article licensed under the terms of the Creative Commons Attribution Non-Commercial License (http://creativecommons.org/licenses/by-nc/3.0/) which permits unrestricted, non-commercial use, distribution and reproduction in any medium, provided the work is properly cited. 